(Coord.) Estudio multidisciplinar del Parque Natural del Montgó (Alicante). Consellería d’Administració Pública. Agéncia del Medi Ambient. 283 p.p.

GALLEGO, M.J. -1999- Lathyrus L. en Castroviejo, $\mathrm{S}$ et al. (éds.). Flora Iberica VII:Leguminosae (partim). Real Jardín Botánico, C.S.I.C. Madrid. HERRANZ, J.M. y C. GÓMEZ-CAMPO -1986Contribución al conocimiento de la flora y vegetación de la comarca de Alcaráz (Albacete). Caja de Ahorros de Albacete. 279 p.p.

MARTINEZ-LABORDE, J.B. -1993- DC. en Castroviejo, S. et al. (éds) Flora Iberica IV: Cruciferae-Monotropaceae. Real Jardín Botánico, C.S.I.C. Madrid.

MATEO, G. y M.B. CRESPO -1995- Flora abreviada de la Comunidad Valenciana. Ed. Gamma. 483 p.p.

MATEO, G. y M.B. CRESPO - 1998- Manual para la determinación de la flora valenciana. Flora Montibérica: Monografías 3:1-495.

MATEO, G. y R. FIGUEROLA -1987- Flora analítica de la Provincia de Valencia. Institució Valenciana d’Estudis i Investigació. Edicións Alfons el Magnànim. IAM 14: 1-386
RIGUAL, A. -1972- Flora y vegetación de la provincia de Alicante. Instituto de Estudios Alicantinos. Alicante.

SOLER, J.X.; B. ROCHET y G. MATEO -1995Fragmenta Chorologica Occidentalia 54795509. Anales Jard. Bot. Madrid 53(1): 113-114.

TALAVERA, S. -1990- Silene L. in Castroviejo, S. et al. (éds); 1990-Flora Iberica II: PlatanaceaePlumbaginaceae (partim). Real Jardín Botánico, C.S.I.C. Madrid.

VALDÉS, B., S. TALAVERA, y E. FERNÁNDEZGALIANO -1987- Flora vascular de Andalucía Occidental, Vol. 2. Ed. Ketres. Barcelona.

Aceptado para su publicación en Octubre de 1999

Dirección del autor. Departamento de Biología Vegetal, U.D. Botánica, Facultad de Ciencias Biológicas, Universitat de València. Dr. Moliner, 50. 46100 Burjassot. (Valencia).

\title{
78. REFERENCIAS COROLÓGICAS DE PLANTAS VASCULARES EN EL NW IBÉRICO
}

\author{
Raquel ALONSO REDONDO, María José LÓPEZ PACHECO, \\ Emilio PUENTE GARCÍA y Ángel PENAS MERINO
}

Geographical distribution references of vascular plants for NW Spain

Palabras clave. Plantas vasculares, corología, NW España.

Key words. Vascular plants, geographical distribution, NW Spain.

En el presente trabajo ofrecemos una relación de plantas herborizadas en la provincia de León, señalando con un asterisco (*) aquellas que son novedad para dicha provincia. 
Los taxones aparecen colocados por orden alfabético y la nomenclatura y la autoría de cada uno de ellos sigue a Tutin et al. (196876), Castroviejo et al. (1990-1997), Morales Valverde (1986) y Galán Cela (1989).

Todo el material testigo se encuentra depositado en el Herbario LEB-Jaime Andrés Rodríguez del Departamento de Biología Vegetal de la Universidad de León.

\section{ASTERACEAE}

*Cirsium echinatum (Desf.) DC.

LEÓN: Pola de Gordón, 30TTN84, ruderal, 4VIII-1977, C. Pérez Morales, LEB 25452 (sub. C. eriophorum (L.) Scop. subsp. chodati (BarbeyGampert) Rivas-Martínez, T.E. Díaz, F. Prieto, J. Loidi \& A. Penas). Localidad que coloca muy al norte la distribución de este taxón para el que Talavera y Valdés (1976) daban un areal típicamente mediterráneo.

*Inula conyza DC. subsp. gutierrezii (Pau) Galán Cela LEÓN: Valle del Díez (Valmartino), 30TUN2840, 1100 m, quejigar, 19-VIII-1997, R. Alonso Redondo, LEB 63539. Valle de la Duerna (prox. de Ocejo de La Peña), 30TUN2846, 1050 m, borde de carretera, 17-VII-1997, R. Alonso Redondo, LEB 63536.

\section{CARYOPHYLLACEAE}

Thelephium imperati L. subsp. imperati

LEÓN: Crémenes, 30TUN25, $1000 \mathrm{~m}$, pedregales calizos en claros del sabinar, 21-VI1998, E. Puente, LEB 63541. Valle del Díez (Valmartino), 30TUN2840, $1205 \mathrm{~m}$, zona pedregosa caliza, 12-IV-1997, R. Alonso Redondo, LEB 63540. Valle de la Duerna (prox. de Ocejo de La Peña), 30TUN2846, $1050 \mathrm{~m}$, pedregal calizo, 14-VIII-1997, R. Alonso Redondo, LEB 63536. Citas que sirven de puenteo entre la de La Vega de los Viejos (Cabrillanes), 29TQH26, de Laínz (1976) y la de Monte Corona (Posada de Valdeón), 30TUN48, de Aedo et al. (1993).

\section{CONVOLVULACEAE}

*Cuscuta approximata Bab. subsp. approximata
LEÓN: Prox. de Valmartino, 30TUN2839, $1065 \mathrm{~m}$, en cardal sobre Lactuca viminea (L.) J. \& C. Presl, 25-VII-1997, R. Alonso Redondo, LEB 63289. Ibid., en pastizales basófilos sobre Astragalus sp., LEB 63291. Villarino del Sil, 30TQG08, camino de bosque mixto sobre Urtica sp., 10-VIII-1981, J. Andrés, LEB 55564.

\section{FABACEAE}

\section{Genista tinctoria L.}

LEÓN: Cerezal, 30TUN3638, 975 m, pastizal, 21-VI-1996, R. Alonso Redondo, LEB 63290. Citada de Llamas de Rueda, 30TUN22, por Carbó et al. (1972). La mención de Borja (1953) de la zona de Nocedo, 30TUN05, no ha podido ser corroborada por nosotros.

\section{Lens nigricans (Bieb.) Godron}

LEÓN: Cistierna, 30TUN2743, $1000 \mathrm{~m}$, canchal de bloques pequeños calizos, 10-V-1996, R. Alonso Redondo, LEB 63285. Robledo de la Guzpeña (Serrones), 30TUN3341, 1190 m, encinar con afloramientos de calizas, 21-VI-1996, R. Alonso Redondo, LEB 62297. Citada de Carucedo, 29TPH80, por Giménez y Amigo (1996).

\section{* Melilotus neapolitana Ten.}

LEÓN: Valle de Nuestra Señora (prox. de Santa Olaja de la Varga), 30TUN2846, $1075 \mathrm{~m}$, pastizal basófilo pedregoso, 16-VI-1997, R. Alonso Redondo, LEB 63287.

\section{Tetragonolobus maritimus (L.) Roth}

LEÓN: Olleros de Alba, 30TTN74, 17-VI1980, F. Llamas, LEB 37963. Valle del Díez (prox. de Valmartino), 30TUN2839, $1100 \mathrm{~m}$, vaguada con quejigos, 31-V-1997, R. Alonso Redondo, LEB 63286. Segunda mención provincial, después de la de Valencia de Don Juan, 30TTM98 (Penas Merino, 1984).

\section{GENTIANACEAE}

\section{Centaurium somedanum Laínz}

LEÓN: Cospedal de Babia, 29TQH4062, 1300 m, pastizal, 26-VII-1992, E. Puente \& Mª J. López, LEB 63537. Nueva mención leonesa de este escaso y vulnerable endemismo ubiñense conocido, únicamente, de La Vega de los Viejos (Cabrillanes), 
29TQH26 (Laínz, 1976) y Cuetalbo (Torre de Babia), 29TQH36 (Romero, 1983), así como de las localidades somedanas asturianas de La Riera (Somiedo), 29TQH28 y Villanueva (Teverga), 29TQH38 (Laínz, 1976) y km 25-26 de la carretera entre Belmonte de Miranda y Pola de Somiedo, cerca del Embalse de La Malva, 29TQH27 (Fernández Prieto, 1978).

\section{LAMIACEAE}

*Thymus $x$ ibericus Sennen \& Pau

(=Th. mastichina (L.) L. subsp. mastichina $\mathrm{x}$ Th. mastigophorus Lacaita)

LEÓN: Puerto del Pando, 30TUN4054, 1420 $\mathrm{m}$, pastizal acidófilo con aportes de calizas, 9-VII1997, R. Alonso Redondo, LEB 63543.

\section{Thymus $x$ sennenii Pau}

(=Th. mastichina (L.) L. subsp. mastichina $\mathrm{x}$ Th. pulegioides L.)

LEÓN: Valmartino, 30TUN2740, $1100 \mathrm{~m}$, tomillar,15-VI-1996, R. Alonso Redondo, LEB 63534. Segunda cita provincial después de la de Geras de Gordón, 30TTN75 (Herrero Cembranos et al., 1988).

*Thymus $x$ viciosoi (Pau) R. Morales

(=Th. pulegioides L. x Th. zygis L. subsp. zygis)

LEÓN: Cruce de Benllera a Carrocera, 30TTN73, $1050 \mathrm{~m}$, rebrote de tomillos en pastizal, 3-VII-1990, E. Puente \& M $^{a}$ J. López, LEB 63535. Taxón en el que se observan caracteres intermedios entre los parentales, como menciona el monógrafo y autor del híbrido Morales Valverde (1986).

\section{PRIMULACEAE}

\section{*Primula farinosa $\mathrm{L}$.}

LEÓN: Base del Espigüete (Valverde de la Sierra), 30TUN5156, $1650 \mathrm{~m}$, comunidad turfófila, 5-VIII-1998, R. Alonso Redondo, LEB 63288. Taxón que confirma la presencia en la provincia de esta especie de la que A. Kress in Castroviejo et al. (1997) dice no haber observado ningún pliego de herbario.

\section{SCROPHULARIACEAE}

*Scrophularia crithmifolia Boiss.
LEÓN: Subida al Espigüete, 30TUN5256, 2050 $\mathrm{m}$, pedregal calizo móvil de bloques pequeños y medianos, 14-VI-1997, R. Alonso Redondo, LEB 63531. Taxón que hemos encontrado citado de varias localidades de la provincia de Palencia (García González, 1990).

\section{BIBLIOGRAFÍA}

AEDO, C., J. J. ALDASORO, J. M. ARGÜELLES, J. L. DÍAZ ALONSO, J. M. GONZÁLEZ DEL VALLE, C. HERRÁ (+), M. LAÍNZ, G. MORENO MORAL, J. PATALLO y O. SÁNCHEZ PEDRAJA - 1993- Contribuciones al conocimiento de la flora cantábrica. Fontqueria 36: 349-374.

BORJA CARBONELL, J. -1953- Datos para la flora cantábrica. Plantas de Nocedo (León). Anales Inst. Bot. Cavanilles 11(1): 419-436.

CARBÓ, R., M. MAYOR, J. ANDRÉS y J. M. LOSA - 1972- Aportaciones al catálogo florístico de la provincia de León. Anal. Fac. Veter. León 18(1): 225-352.

CASTROVIEJO, S., M. LAÍNZ, G. LÓPEZ GONZÁLEZ, P. MONTSERRAT, F. MUÑOZ GARMENDIA, J. PAIVA y L. VILLAR (eds.) 1990- Flora Iberica. Vol II. Serv. Publ. CSIC. Real Jardín Botánico. Madrid.

CASTROVIEjo, S., C. AEDO, M. LAínZ, R. MORALES, F. MUÑOZ GARMENDIA, G. NIETO FELINER y J. PAIVA (eds.) -1997Flora Iberica. Vol V. Serv. Publ. CSIC. Real Jardín Botánico. Madrid.

FERNÁNDEZ PRIETO, J. A. -1978- Notas sobre la flora somedana. Notas florísticas y ecológicas sobre la flora ibérica. II: 1-5.

GALÁN CELA, P. -1989- Tipificación de dos nombres de Pau y una nueva combinación en el género Inula. Fontqueria 17: 9-10.

GARCÍA GONZÁLEZ, M. E. -1990- Flora y vegetación de la Sierra del Brezo y de la comarca de La Peña (Palencia). Microficha 54. Ser. Publ. Universidad de León.

GIMÉNEZ DE AZCÁRATE CORNIDE, J. y J. AMIGO VÁZQUEZ - 1996- Inventario da flora vascular de afloramientos calios de Galicia (Pteridophyta e Spermatophyta). Cadernos da Area de Ciencias Biolóxicas (Inventarios) do Seminario de Estudos Galegos. Vol. XII. 181 
pp. A Coruña.

HERRERO CEMBRANOS , L., M. E. GARCÍA GONZÁLEZ y A. PENAS MERINO -1988- De Plantis legionensibus. Notula XI. Acta Bot. Malacitana 13: 319-323.

LAÍNZ, M. -1976- Aportaciones al conocimiento de la flora cántabro-astur, XI. Bol. Inst. Est. Ast., ser. C, 22: 3-44.

MORALES VALVERDE, R. - 1986- Taxonomía de los géneros Thymus (excluida la Sección Serpyllum) y Thymbra en la Península Ibérica. Ruizia 3: 5-324.

PENAS MERINO, A. -1984- Nuevos taxones para la flora leonesa. Lagascalia 13(1): 3-16.

ROMERO RODRÍGUEZ, C. M. -1983- Flora y vegetación de la cuenca alta del río Luna (León). ICONA Monografías 29: 5-273.

TALAVERA, S. y B. VALDÉS -1976- Revisión del género Cirsium (Compositae) en la Península Ibérica. Lagascalia 5(2): 127-223.
TUTIN, T. G., V. H. HEYWOOD, N. A. BURGES, D. M. MOORE, D. H. VALENTINE, S. M. WALTERS \& D. A. WEBB (eds.). -1968-76Flora Europaea. Vol. II, III, IV. Cambrigde University Press.

Aceptado para su publicación en Abril de 1999

Dirección de los autores. Departamento de Biología Vegetal. Universidad de León. Campus de Vegazana s/n. E-24071. León. España.

\title{
79. NUEVAS LOCALIDADES ALICANTINAS DE CARDUNCELLUS DIANIUS WEBB
}

\author{
María Rosa SOLER, José Carlos CRISTÓBAL y Manuel B. CRESPO
}

New alicantine localities of Carduncellus dianius Webb

Palabras clave: Corología, flora, Alicante, España.

Key words: Chorology, flora, Alicante, Spain.

Durante la realización de diversos estudios sobre la flora y la vegetación de las sierras del norte de la provincia de Alicante, se ha constatado la existencia en la Serra de Bèrnia de dos nuevas localidades del interesante endemismo diánico-pitiúsico Carduncellus 